\title{
Nets with collisions (unstable nets) and crystal chemistry
}

\section{Olaf Delgado-Friedrichs, Stephen T. Hyde, Shin-Won Mun, Michael $\mathrm{O}^{\prime}$ Keeffe and Davide M. Proserpio}

Acta Cryst. (2013). A69, 535-542

Copyright (C) International Union of Crystallography

Author(s) of this paper may load this reprint on their own web site or institutional repository provided that this cover page is retained. Republication of this article or its storage in electronic databases other than as specified above is not permitted without prior permission in writing from the IUCr.

For further information see http://journals.iucr.org/services/authorrights.html

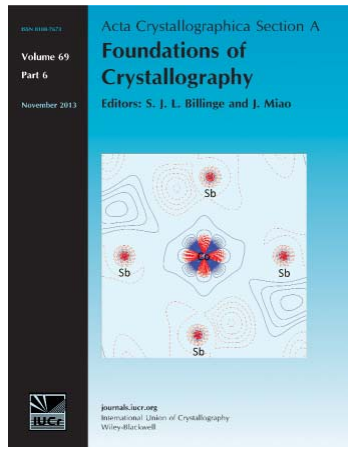

Acta Crystallographica Section A: Foundations of Crystallography covers theoretical and fundamental aspects of the structure of matter. The journal is the prime forum for research in diffraction physics and the theory of crystallographic structure determination by diffraction methods using X-rays, neutrons and electrons. The structures include periodic and aperiodic crystals, and non-periodic disordered materials, and the corresponding Bragg, satellite and diffuse scattering, thermal motion and symmetry aspects. Spatial resolutions range from the subatomic domain in charge-density studies to nanodimensional imperfections such as dislocations and twin walls. The chemistry encompasses metals, alloys, and inorganic, organic and biological materials. Structure prediction and properties such as the theory of phase transformations are also covered.

\section{Crystallography Journals Online is available from journals.iucr.org}


Acta Crystallographica Section A

Foundations of Crystallography

ISSN 0108-7673

Received 5 June 2013

Accepted 25 July 2013
C 2013 International Union of Crystallography Printed in Singapore - all rights reserved

\section{Nets with collisions (unstable nets) and crystal chemistry}

\author{
Olaf Delgado-Friedrichs, ${ }^{a}$ Stephen T. Hyde, ${ }^{a}$ Shin-Won Mun, ${ }^{b}$ Michael O'Keeffe ${ }^{b, c *}$ \\ and Davide M. Proserpio ${ }^{\mathrm{d}, \mathrm{e}}$
}

aDepartment of Applied Mathematics, Research School of Physics, Australian National University, Canberra, ACT 0200, Australia, ${ }^{\mathbf{b}}$ Graduate School of EEWS(WCU), KAIST, 373-1, Guseng Dong, Yuseong Gu, Daejeon 305-701, Republic of Korea, ' Department of Chemistry and Biochemistry, Arizona State University, Tempe, AZ 85287, USA, 'dUniversità degli Studi di Milano, Dipartimento di Chimica, Via Golgi, 19, 20133 Milano, Italy, and ${ }^{\mathbf{e}}$ Samara Center for Theoretical Materials Science (SCTMS), Samara State University, Ac. Pavlov St 1, Samara 443011, Russia.

Correspondence e-mail: mokeeffe@asu.edu

Nets in which different vertices have identical barycentric coordinates (i.e. have collisions) are called unstable. Some such nets have automorphisms that do not correspond to crystallographic symmetries and are called non-crystallographic. Examples are given of nets taken from real crystal structures which have embeddings with crystallographic symmetry in which colliding nodes either are, or are not, topological neighbors (linked) and in which some links coincide. An example is also given of a crystallographic net of exceptional girth (16), which has collisions in barycentric coordinates but which also has embeddings without collisions with the same symmetry. In this last case the collisions are termed unforced.

\section{Introduction}

In this paper we are concerned with periodic nets which are simple connected periodic graphs [see e.g. Delgado-Friedrichs \& O'Keeffe (2005) for terminology and definitions] often encountered in the analysis of the topology of crystal structures. It is convenient at the outset to distinguish between abstract nets and their Euclidean embeddings (Chung et al., 1984). Specifically in this paper the vertices and edges of the graph correspond to nodes and links in an embedding. One reason for doing this is that we want to refer to the length of a link, whereas the 'length' of a single edge of a graph has no meaning.

The classical nets of crystal chemistry are mostly the nets of sphere packings, i.e. they have embeddings in which all links are of equal length and are the shortest distances between nodes. The net of diamond is a familiar example. The taxonomy of such nets and their relevance to materials design are now reasonably well established (Delgado-Friedrichs et al., 2007) at least for those nets which admit tilings. However, particularly as the synthesis of metal-organic frameworks (MOFs) has developed, many nets have been discovered that do not admit tilings because many of the strong rings (cycles that are not the sum of smaller cycles) are catenated with other rings (Delgado-Friedrichs et al., 2005). A recent example of a crystal structure based on a net in which every ring is catenated with another is provided by $\mathrm{Yu}$ et al. (2012). In the present paper we call attention to another class of nets, so far little discussed, in which different nodes are at the same site in high-symmetry embeddings. Some of these are also nets with entangled strong rings.

A very useful approach to analysis of periodic nets is to first compute a placement in which one vertex is arbitrarily assigned coordinates (e.g. 0,0,0) and the rest then assigned barycentric coordinates that are the average of those of their neighbors. It is easy to show that this results in a unique set of coordinates (Delgado-Friedrichs, 2005). These are unit-cell coordinates in the usual crystallographic sense and are independent of any metric associated with the size and shape of the unit cell; for this reason one refers to a placement rather than an embedding. The idea appeared early in the work of Tutte (1960,1963) on finite graphs. The resulting set of coordinates for periodic graphs has been called a standard realization by Kotani \& Sunada (2000) and an equilibrium placement by Delgado-Friedrichs (2004, 2005). These authors show that the coordinates are those that would result at equilibrium (minimum energy) for any finite fixed unit-cell volume if the links of the net were replaced by uniform harmonic springs. As discussed below, for certain graphs two or more nodes have identical barycentric coordinates and we say that such a graph has collisions. Graphs without collisions were called stable by Delgado-Friedrichs (2005). Barycentric coordinates are central to the algorithms of Systre, a computer program for determining the symmetry and identity of periodic nets (Delgado-Friedrichs \& O'Keeffe, 2003) and which was used to determine symmetries in this work.

Recently we have called attention to a class of nets with collisions that nevertheless have crystallographic symmetry 
(de Campo et al., 2013; see also Eon, 2011). This raises the questions as to what are the requirements for a graph with collisions to be crystallographic and whether such nets are relevant to crystal chemistry. The latter question is addressed in this paper. We do not discuss nets known to have noncrystallographic symmetry ('NC nets') which have been the topic of several recent publications (Moreira de Oliveira Jr \& Eon, 2011, 2013). Nor do we discuss the possible symmetries of embeddings with all finite links of unstable graphs; that topic has also recently been addressed by Thimm (2009) and by Eon (2011).

We have occasion below to refer to the quotient graph of a net. This is the finite graph with all translationally equivalent points merged into one, and the edges labeled to indicate the adjacencies of vertices (Chung et al., 1984).

We will find examples of different graphs that have embeddings with identical arrays of nodes and links (i.e. they coincide as sets of points and lines). Following a suggestion of Grünbaum (2003) we refer to such structures as isomeghethic.

Net symbols of the sort abc or abc-d are RCSR (Reticular Chemistry Structure Resource) symbols (O'Keeffe et al., 2008) and data for them are available at the RCSR web site (http://rcsr.anu.edu.au/). That site also reports vertex symbols (Blatov et al., 2010). The nets discussed here generally have very high topological density (O'Keeffe, 1991). The RCSR reports $\mathrm{TD}_{10}$ which is the average over all vertices of the number of vertices reachable from each vertex in paths of 0 to 10 edges. Some also have exceptional girth (called 'smallest ring' in the RCSR). Nets for which the shortest rings at every angle are equal are called uniform by Wells (1977), who attached special importance to them in crystal chemistry. Several of the nets in this paper are uniform.

\section{Polygons and polyhedra with collisions}

It is instructive to first consider some finite structures with collisions between vertices. Specifically an abstract $p$-gon (a polygon with $p$ vertices) is a simple cycle with $p$ vertices and $p$ edges. The regular polygons were long ago generalized from those with embeddings as a convex figure with symbol $\{p\}$ to include the star polygons $\{p / n\}$ (illustrated in Fig. 1 for $p=8$ ). Usually $p$ and $n$ are considered co-prime but, as Grünbaum (2003, 2007) points out, for complete generality one should

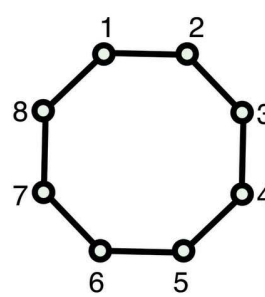

$\{8\}$

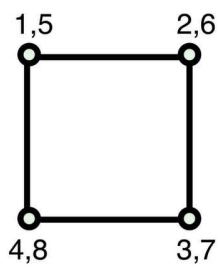

$\{8 / 2\}$

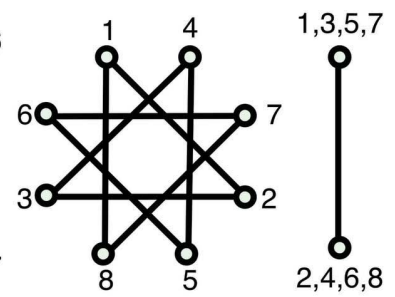

$\{8 / 3\}$
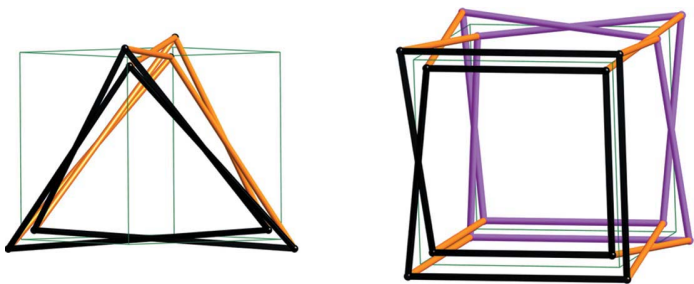

Figure 2

Left, the polyhedron $\{6 / 2,3\}$. One hexagonal face is in black. Right, the polyhedron $\{8 / 2,3\}$. Two octagonal faces are shown in black and magenta, respectively.

really take all cases of $n \leq p / 2$ even though nodes become coincident when $p$ and $n$ are not co-prime.

These generalized polygons can serve as the faces or vertex figures of regular polyhedra with Schläfli symbol $\{h, k\}$ in which faces are $\{h\}$ and the vertex figures are $\{k\}$. The Platonic solids are $\{3,3\},\{3,4\},\{4,3\},\{3,5\}$ and $\{5,3\}$. Adding star polygons one gets the regular icosahedral Kepler-Poinsot polyhedra $\{5 / 2,3\},\{3,5 / 2\},\{5 / 2,5\}$ and $\{5,5 / 2\}$. Grünbaum adds to these regular polyhedra such as $\{6 / 2,3\}$ and $\{8 / 2,3\}$ (see Fig. 2), $\{12 / 3,3\}$ and so on indefinitely. $\{6 / 2,3\}$ is the same combinatorially as the cube $\{4,3\}$, a fact that emphasizes that the cube can also be considered as having intersecting faces that are skew hexagons (Petrie polygons, see e.g. O'Keeffe, 2008) so $\{6 / 2,3\}$ does not have a maximum-symmetry embedding with coincident nodes.

\section{Non-crystallographic and crystallographic nets with collisions}

Perhaps the simplest case of occurrence of collisions in nets is when two vertices have the same neighbors (Moreira de Oliveira Jr \& Eon, 2013). Clearly there is a net automorphism that simply involves interchanging those two vertices leaving the rest fixed. This cannot correspond to a crystallographic symmetry operation which acts on rigid bodies. Such nonrigid-body symmetries can lead to complicated automorphism groups, as is well documented for non-rigid molecules (Longuet-Higgins, 1963). They are sometimes called 'local symmetries' but this term is also used to refer to short-range spatial arrangements in crystals. Moreira de Oliveira Jr \& Eon (2013) call them 'bounded automorphisms'. A common occurrence of such collisions in the graph of a chemical structure is found in the familiar copper carboxylate paddlewheel shown in Fig. 3. Usually in determining the underlying
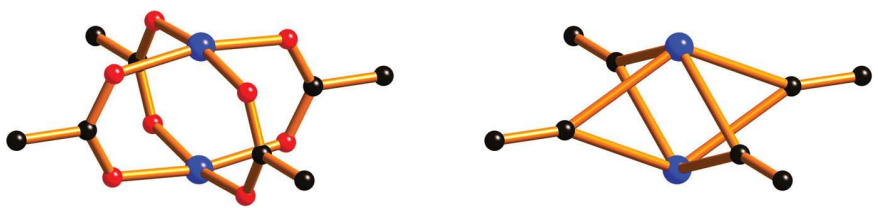

Figure 3

Left, part of a structure with the paddlewheel secondary building unit. Carbon atoms black, oxygen red and copper blue. On the right the -Olinks shown as single links and the two $\mathrm{Cu}$ atoms linked to the same four $\mathrm{C}$ atoms.

The regular octagons. In each case the edges are 1223344556677881 . 

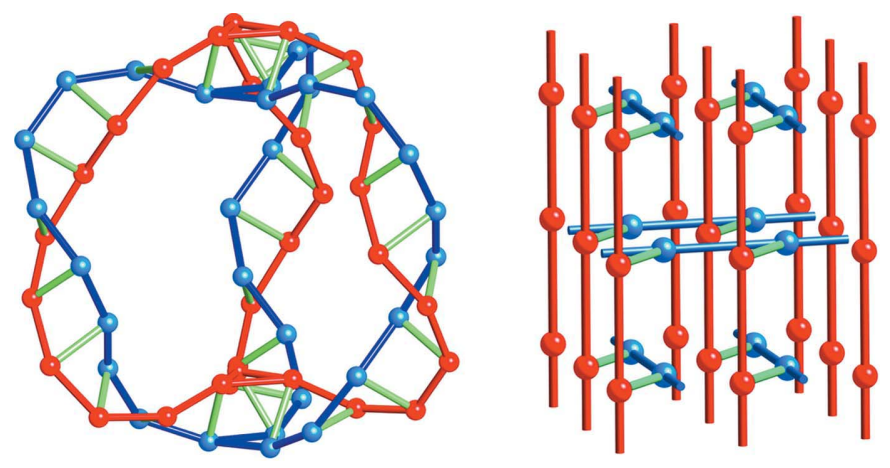

Figure 4

Left, a fragment of a 3-periodic ladder structure (the 'rungs' are green). Nodes are all 4-coordinated as shown near the top and bottom. Right, an 'antiladder' structure from de Campo et al. (2013). Rungs are again green. Red and blue nodes collide and the resulting structure is tetragonal $\left(P 4_{2} / m m c\right)$ isomeghethic with cds.

topology of a molecule or crystal, 2-coordinated (2-c) nodes such as a linking -O- atom are subsumed into edges so that both $\mathrm{Cu}$ atoms are considered directly linked to the same four carboxylate $\mathrm{C}$ atoms (the points of extension of the copper secondary building unit). The usual procedure at this point in determining a net is simply to replace the two $\mathrm{Cu}$ atoms with one 4-c node.

A generalized ladder is obtained when a finite number (two or more) of identical interpenetrating nets are joined by extra links in such a way that the nodes connected by the extra links are in identical orientation. In such a situation there are automorphisms of the whole structure that map any copy of the component nets to any other copy while not changing their orientation in space. If these automorphisms were to be taken as crystallographic operations they would have to be translations, but because they are a finite set that is clearly impossible. An example of a 3-periodic ladder is shown in Fig. 4. The embedding comes from a sphere packing with an embedding in $I 4_{1} 32$. This is the sphere packing $4 / 3 / c 26$ of Fischer (1974) with RCSR symbol uld-z. ${ }^{1}$

On the other hand if all colliding nodes were linked to different nodes, that did not themselves collide with each other, they can be distinguished and symmetry determined. Thus two or more component subnets could come together without links superimposing. We call these antiladders. A simple example is shown in Fig. 4 in which the components are rods running in orthogonal directions. This example is in fact the net called 4(3)5, one of the crystallographic minimal nets with collisions discussed by de Campo et al. (2013) and Eon (2011).

The process of distinction of nodes by their neighbors can be extended to further neighbors, but after next-nearest neighbors the procedure becomes rather complex to implement in practice. Indeed, the computer program Systre, which is the major source of symmetry information for periodic

\footnotetext{
$\mathbf{1}$ The net of this sphere packing is the same graph as that of sphere packing $4 / 3 / c 25$ but the two embeddings (uld and uld-z in the RCSR) are not ambient isotopic (cannot be transformed from one to the other without breaking and reforming links) (see Moreira de Oliveira Jr \& Eon, 2013).
}

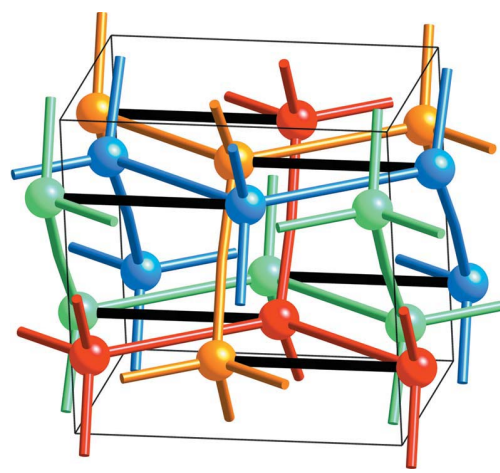

Figure 5

Four interpenetrating diamond nets joined by a fifth link, black. Note that the red and blue nets are not directly linked, nor are the yellow and green ones. This is net rld-z.

graphs, gives up at the point where next-nearest neighbors have collisions. In all the unstable nets discussed in this paper colliding vertices are distinguishable as described above, and Systre reports a crystallographic symmetry and an embedding in that symmetry which is used in this work.

It should be remarked that Systre only looks for automorphisms of the net that are consistent with the given periodic structure, i.e. a translation group $T$. In principle it is still possible that other automorphisms could occur as described by Moreira de Oliveira Jr \& Eon (2011) and we cannot at present prove their absence mathematically. To allow for this eventuality we say that our nets are T-crystallographic and refer to the symmetry determined by Systre as the Systre symmetry. However our main purpose is simply to get a crystallographic symmetry so we can present an embedding with collisions required by that symmetry.

\section{Nets with zero-length links in maximum-symmetry embeddings}

\subsection{A net based on interpenetrating diamond nets}

We (de Campo et al., 2013) earlier called attention to the fact that, although all the minimal (genus 3) nets had crystallographic symmetry (Eon, 2007), seven of them had collisions in their maximum-symmetry embeddings. In those embeddings they were isomeghethic to one of the minimal nets without collisions. We have not yet identified any of these minimal nets with collisions as the topologies of real crystal structures. However, an interesting structure reported by Montney et al. (2007) does have an underlying topology of this type. In the structure (2) of those authors, four interpenetrating 4-c dia (diamond) nets are joined into one continuous network by a fifth link between pairs of nodes. An illustration with symmetry Pbcn (the same as the observed crystal structure) is in Fig. 5. The net has the interesting property that each dia subnet is directly joined to only two of the three others and in fact can also be drawn as two interpenetrating dia nets joined by a fifth link as shown in Fig. 6. In that drawing links partly overlap; however, in the limit of a full symmetry embedding, one link has zero length and nodes 
Figure 6

A fragment of two interpenetrating dia nets linked by a short fifth link. Note that the only links are between nodes colored red and blue.

coincide in pairs. The Systre symmetry is $\operatorname{Im} \overline{3} m$ with both vertices at $0,0,0$ so it is in fact isomeghethic with the net of the nodes of the body-centered lattice with shortest lattice vectors as links, RCSR symbol bcu. Another notable feature of the structure is that all the rings are 6-rings with three at each angle so 30 meeting at each vertex (compare 12 meeting at each vertex for dia). In the RCSR the Pbcn embedding is given with the symbol rld-z. The extension $\mathbf{- z}$ indicates that the embedding is not a maximum-symmetry one. In that embedding all links are equal length and do not intersect or overlap; it is an interesting question whether this is the highestsymmetry embedding with this property.

\subsection{Other nets derived from linked interpenetrating pairs of diamond nets}

Linked pairs of diamond nets give rise to a number of other interesting topologies. Fig. 7 illustrates the most symmetrical $(P n \overline{3} m)$ embedding of a pair of interpenetrating diamond nets. This has been known in crystal chemistry for 100 years as the structure of the $\mathrm{O}$ atoms of cuprite, $\mathrm{Cu}_{2} \mathrm{O}$, with $-\mathrm{Cu}-$ acting as links (Bragg \& Bragg, 1915). Connecting the two vertices in the unit cell gives a 5-c stable net with symmetry $R \overline{3} m$ with RCSR symbol fnu (Fig. 7). This net also has all 6-rings, now with 48 meeting at each vertex. It might be noted that, although many of those rings are catenated with other rings as they come from catenated dia nets, not all are catenated and this net does admit a tiling (Blatov et al., 2007).

If only half the vertices were linked to produce a $(4,5)$-c net as shown in Fig. 8, then one gets again a net with collisions (data given in the RCSR as sld-z). The pairs of newly linked
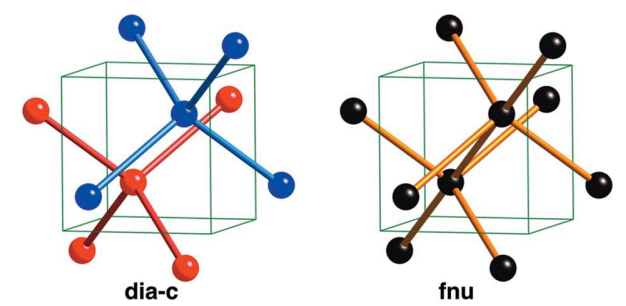

Figure 7

Left, the cubic $(P n \overline{3} m)$ cell of two interpenetrating dia nets. Right, a primitive rhombohedral $(R \overline{3} \mathrm{~m})$ cell of fnu. The extra link is along [111] of that cell.

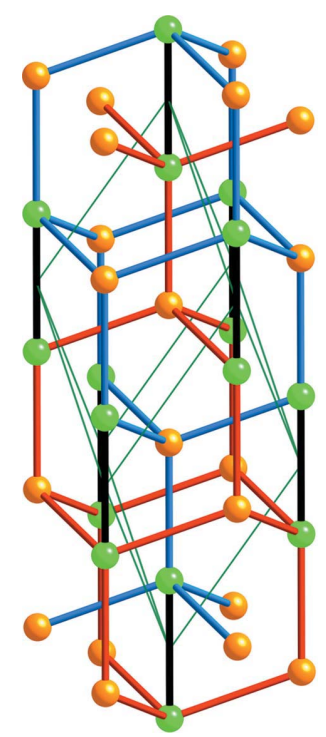

Figure 8

An equal-link embedding (sld-z) of a (4,5)-c net with collisions. Red and blue links outline dia nets.

vertices collide and now one gets a structure isomeghethic with the fluorite net, flu, and with symmetry $F m \overline{3} m$, pairs of 5-c vertices at $0,0,0$ and single 4 -c vertices at $\frac{1}{4}, \frac{1}{4}, \frac{1}{4}$. A double interpenetrated sld-z is observed for a viologen $\mathrm{Zn}$ complex (Tan et al., 2012), although the authors did not discuss it in these terms.

If, alternatively, nodes of pairs of diamond nets were linked so that previously unlinked nodes in the same orientation are linked, then in barycentric coordinates two copies of the same structure, including links, are superimposed. Accordingly the structure is a (non-crystallographic) ladder.

Fig. 9 shows quotient graphs for the diamond-related nets.
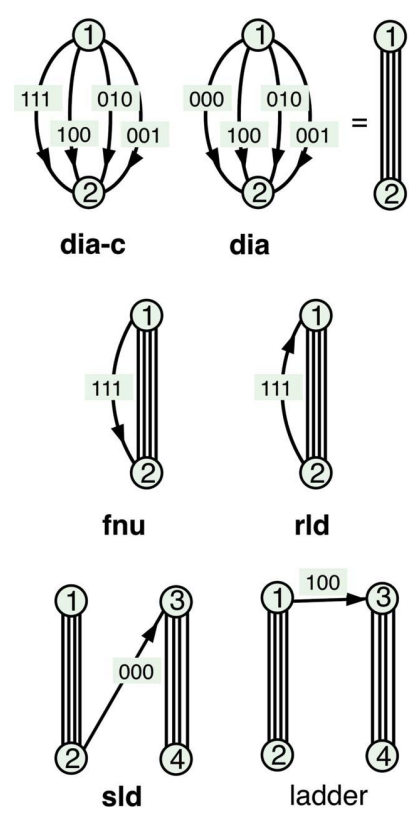

Figure 9

Quotient graphs for nets with dia nets as sub-graphs. 


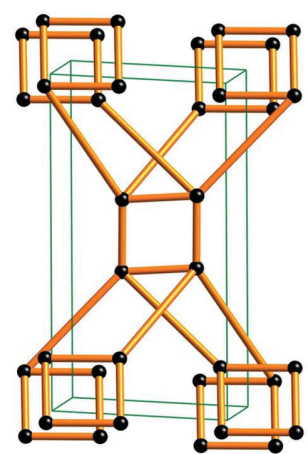

(a)

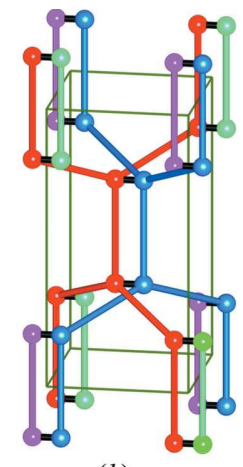

(b)

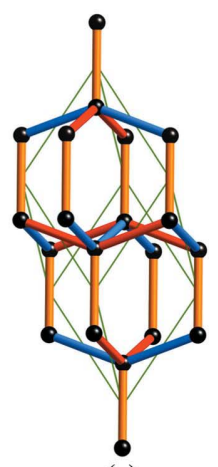

(c)
Figure 10

(a) An embedding of the net hxc using the coordinates of the nodes in the crystal structure. (b) An embedding with short links (black). In the maximum-symmetry embedding those links have zero length. Different color links belong to separate ths nets. (c) A primitive cell of the sqp net isomeghethic with the maximum-symmetry embedding of hxc.
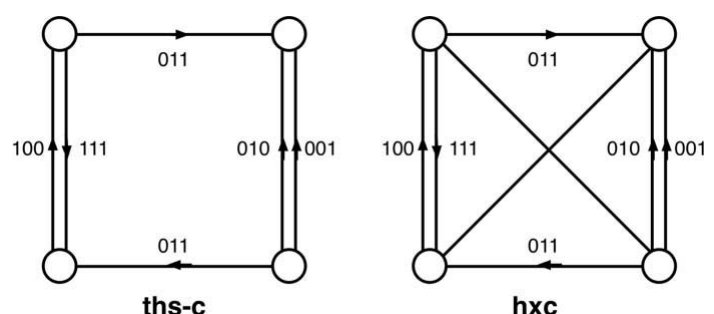

Figure 11

Quotient graphs for ths-c4 and hxc. The graph on the right has the same labeling as that on the left but with two extra edges (shown as diagonal lines with labels 000).

\subsection{A net with collisions related to the ths net}

Our next net, RCSR symbol hxc, was found in a copper imidazolate MOF by Huang et al. (2004). Long and shorter ditopic linkers combine to link $\mathrm{Cu}$ atoms into a uninodal 4-c net as shown for the real structure with symmetry $P 2_{1} / n$ in Fig. 10. This net has vertex symbol $4.8_{4} .8_{4} .8_{6} .8_{4} .8_{6}$, i.e. 248 -rings meeting at each vertex, and is rather dense $\left(\mathrm{TD}_{10}=1918\right.$, compare 981 for dia). The structure has collisions in the Systre symmetry of $I 4 / \mathrm{mmm}$ and pairs of nodes in $0,0, z$ with $z \sim$ 0.18 . Now 4-rings collapse so both links and nodes collide as shown in the figure. The figure also illustrates that again the net can be considered as four linked subnets, that are now the 3-c minimal net ths (rather than the 4-c dia in the previous example), joined by zero-length links. There are just four nodes in the primitive cell - the same as for ths and ths-c4 (four interpenetrating ths nets) and the structure is isomeghethic with the 5-c net sqp, also shown in Fig. 10.

It is interesting that the quotient graph for four interpenetrating ths nets can be labeled in such a way that additionally linking two pairs of vertices with edges labeled $0,0,0$ produces the quotient graph for hxc (Fig. 11).

\subsection{A net with collisions related to the srs net}

A structure of two linked srs nets was recently reported by Wang et al. (2013). In that work it was found that a copper

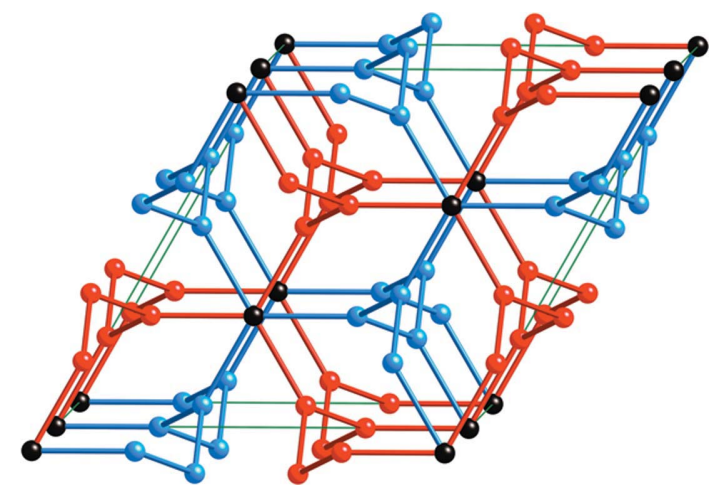

Figure 12

Two srs nets (red and blue) of opposite hand colliding at the nodes shown in black.

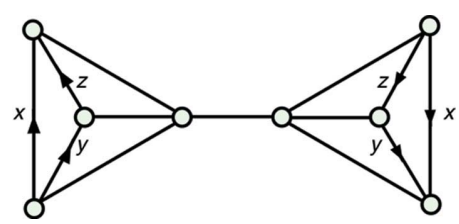

Figure 13

The quotient graph of the net shown in Fig. 12.

cyanide MOF formed two interpenetrating 3-c nets with the srs topology with opposite hand. srs, like ths of the previous example, is a minimal net as it has the minimal number (four) of 3-c vertices in the repeat unit. One fourth of the nodes in the nets are $\mathrm{Cu}$ atoms which form $\mathrm{Cu}-\mathrm{Cu}$ links between the two nets. In barycentric coordinates these pairs of vertices collide and the Systre symmetry becomes $R \overline{3} \mathrm{~m}$. For the two nets to have the exact shape of the cubic srs net the 3-c vertices are at $\frac{1}{4}, 0,0$ and the 4 -c vertices collide in linked pairs at $0,0,0$. For unit link length the unit-cell parameters are $a=4, c=6^{1 / 2}$. This structure is illustrated in Fig. 12. The net has an embedding as a sphere packing (links equal and the shortest distance between nodes) in $R \overline{3}$ with $a=4.000, c=2.4495$, 4-c nodes at 0 , $0.0,0.2041$ and $3-c$ nodes at $0.0000,0.2500,0.2041$. The vertex symbol is $\left(8 \cdot 8 \cdot 10_{6}\right)_{3}\left(8_{2} \cdot 10_{5} \cdot 8_{2} \cdot 10_{5} \cdot 8_{2} \cdot 10_{5}\right.$.). This is assigned RCSR symbol llw-z.

The quotient graph is just a linked pair of graphs $K_{4}$ (the complete graph with four vertices) as shown in Fig. 13.

\section{A net with collisions but with all links of non-zero length}

A less common occurrence is that of nets in which at maximum-symmetry embedding nodes collide but all links have finite length. Indeed we are aware of just one example from a crystal structure (Ma et al., 2013). Linking $\mathrm{Cu}_{2}\left(-\mathrm{CO}_{2}\right)_{4}$ paddlewheels with a very non-planar tritopic carboxylate linker gave a new edge-transitive $(3,4)$-c net with RCSR symbol mhq. The structure is uniform of girth 12 - the vertex symbol is $\left(12_{15} \cdot 12_{15} 12_{15}\right)_{4}\left(12_{6} \cdot 12_{6} \cdot 12_{12} \cdot 12_{12} \cdot 12_{12} \cdot 12_{12}\right)_{3}$. As Ma et al. discuss, the structure is topologically very dense and the 12-rings catenated to a remarkable degree. 
The crystal structure has symmetry $F 432$ and an embedding of the net in that symmetry is obtained with the 4-c vertex at $x_{1}, 0,0$ and the 3-c vertex at $x_{2}, x_{2}, x_{2}$ with links from $x_{1}, 0,0$ to $\frac{1}{2}-x_{2}, x_{2}, \frac{1}{2}+x_{2}$ as shown in Fig. 14 (drawn with $x_{1}=0.25$ and $x_{2}$ $=0.3$ ). However the Systre symmetry is the supergroup $P 432$ with $\mathbf{a}^{\prime}=\mathbf{a} / 2$ and the 3-c vertices merge in groups of four to a common coordinate set as suggested in the figure. The structure specification in $P 432$ is 4-c vertex at $\frac{1}{2}, 0,0$ and four 3-c vertices at $0,0,0$ with edges from $0,0,0$ to $\frac{1}{2}, \frac{1}{2}, 0$; note however the ambiguity in this description - a point at $0,0,0$ has 12 neighbors symmetry-related to $\frac{1}{2}, \frac{1}{2}, 0$ and is isomeghethic to the $(4,12)$-c net ftw. Accordingly the net topology is best specified either by its lower-symmetry embedding or its quotient graph (see below).

The net is interesting in that there are embeddings in $F 432$ with links of arbitrarily large length (measured in units of the unit-cell edge length $a$ ). Thus links from $\frac{1}{2}-x_{2}, x_{2}, \frac{1}{2}+x_{2}$ to $i+$ $x_{1}, j, k$ with $i, j$ and $k$ integers, together with symmetry-related links, are always embeddings of the same graph. So are links from the same reference node $\left(\frac{1}{2}-x_{2}, x_{2}, \frac{1}{2}+x_{2}\right)$ to $i, j, k+x_{1}$. However links, again from the same reference node, to $i, j+x_{1}$, $k$ generally produce multiple copies of the same net in a complicated way. For example a link to $0, x_{1}, k$ produces $2^{3} .(k-1)^{3}$ interpenetrating $\mathbf{m h q}$ nets. ${ }^{2}$

We call attention to yet another aspect in which the mhq net is special. It and the edge-transitive $(3,4)$-c net bor are the only two such nets known that have as quotient graph the complete bipartite graph $K_{3,4}$. These two quotient graphs are shown in Fig. 15.

Finally we note that mhq has the largest girth (12) of any known edge-transitive 3-periodic graph without 2-c vertices.

\section{A second net with collisions and no zero-length links}

Here we discuss another net that describes a crystal structure (Volkringer et al., 2009). ${ }^{3}$ The structure contains $\mathrm{Mg}_{2} \mathrm{O}_{3}\left(\mathrm{CO}_{2}\right)_{4}$ secondary building units (SBUs), joined to four tritopic linkers and further linked to two other SBUs by a second ditopic linker. Accordingly we abstract the topology as a $(3,6)$-c net known as 3,3,6T26 in the TOPOS database (Blatov, 2006). In barycentric coordinates unlinked nodes collide in pairs. Another pair come close to colliding in barycentric coordinates but if an equal link-length constraint is applied to the embedding they also come together in pairs. In the crystal with symmetry $R \overline{3}$ there are two distinct SBU centers (6-coordinated) at nodes 1 and 2 in general positions $18 f$ (36 nodes). The linkers are of four topological types with 3-c nodes 3 and 4 in position $18 f$ ( 36 nodes) and 5 and 6 at positions $6 c: 0,0, z(12$ nodes) as shown in Fig. 16. In the Systre symmetry, $R \overline{3} \mathrm{~m}$, embedding nodes 1 and 2 jointly occupy general positions $36 i$ and so do nodes 3 and 4 . However, nodes 5 and 6 doubly occupy $6 c: 0,0, z$. The site symmetry is $3 m$ and links to the 6 -c

\footnotetext{
${ }^{2}$ Systre can be used to determine the number of interpenetrating nets. To give a somewhat extreme example: using the operations of $F 432$ a link from $0.2,0.3$, 0.8 to $100.25,100.0,100.0$ produces a single copy of $\mathbf{~ m h q}$; if the link is instead to $100.0,100.25,100.0$ the number of copies of $\mathbf{m h q}$ is $63044792\left(=2^{3} .199^{3}\right)$.

${ }^{3}$ Our analysis differs from that of the original authors.
}
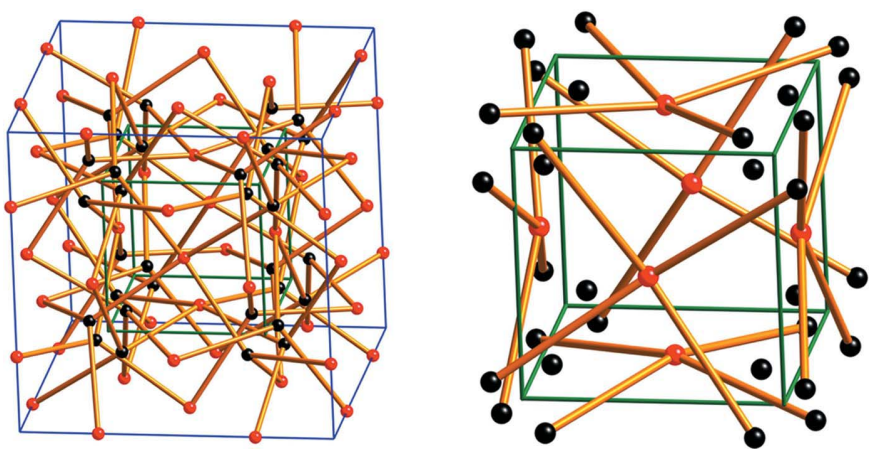

Figure 14

Left, the net mhq in an F432 embedding. The unit cell is outlined in blue. Right, an enlargement of the center of the figure on the left. At maximum symmetry (P432) groups of four black nodes collide and the new unit cell is that shown with green lines.
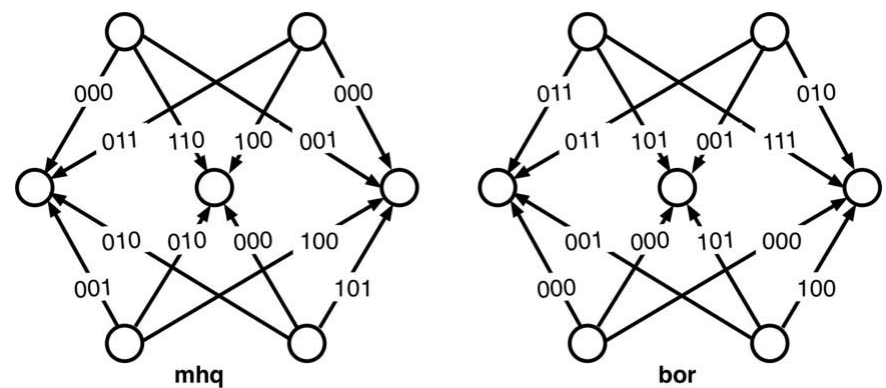

Figure 15

Quotient graphs for two edge-transitive $(3,4)$-c nets. The graphs are the complete bipartite graph $K_{3,4}$.

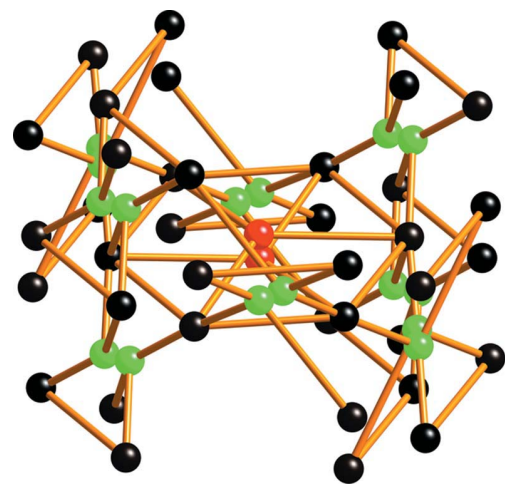

Figure 16

An embedding in $R \overline{3}$ of the $(3,6)$-coordinated net of the crystal structure of Volkringer et al. (2009). The structure illustrated is close to equal links. In barycentric coordinates the red nodes collide in pairs. In an equal-link embedding both the red and green nodes collide in pairs.

node in general positions must be sixfold [i.e. two sets of three from identical positions in a (001) plane related by vertical mirrors].

In the equal-link embedding nodes 3 and 4 come together at $18 h: x, 2 x, z$. But this last is not a collision in the sense used in this paper as the coordinates are not now barycentric.

\section{A net of exceptional girth and unforced collisions}

The question of the largest girth for periodic nets with a given number of vertices in the quotient graph appears to be an 
interesting one that has not yet received much attention. We know of only one net of girth $>13$ that has been found in crystal structures. This is oft $-\mathrm{a}$ uniform binodal 3-c net with vertex symbol $\left(14_{8} \cdot 14_{8} \cdot 14_{8}\right)_{3}\left(14_{4} \cdot 14_{8} \cdot 14_{8}\right)$ which has been found in several materials (Liu et al., 2008, 2010).

The largest girth periodic net we are aware of is a uninodal, edge-2-transitive, 3-c one of girth $16 .^{4}$ This was generated from the uniform large-girth 4-c net ten (Delgado-Friedrichs et al., $2005)$ which has vertex symbol $10_{7} \cdot 10_{7} \cdot 10_{9} \cdot 10_{13} \cdot 10_{12} \cdot 10_{12}(60$ 10-rings at each vertex). Deletion of one edge (edge E2 in RCSR) produces a new 3-c net (Blatov, 2007). This net, with RCSR symbol sxt, has vertex symbol $16_{8} \cdot 16_{.8} \cdot 16_{8}$. Interestingly the stable net ten has symmetry $I 23$ with nodes in general positions $24 f$ with only trivial site symmetry. On the other hand sxt has the Systre supergroup symmetry I432 with vertices now in $24 i$ : $\frac{1}{4}, y, \frac{1}{2}-y$ and point symmetry 2 . In barycentric coordinates $y=\frac{1}{4}$ and nodes collide in groups of three at $8 c: \frac{1}{4}, \frac{1}{4}, \frac{1}{4}$. That site has symmetry 32 but the individual nodes only have symmetry 2 . We call these unforced collisions because there are embeddings without collisions in the same symmetry but with $y \neq \frac{1}{4}$. An embedding with $y=0.292$, a value that makes all links equal in length, is shown in Fig. 17. We remark that, unless forced by symmetry, barycentric coordinates are rarely used for embeddings of nets. In the RCSR for example, the embeddings are, when possible, for equal links, and then subject to that restraint, for minimal density.

\section{Concluding remarks}

We have shown by example that $T$-crystallographic nets with collisions in barycentric coordinates indeed occur in crystal structures. In some cases these collisions are unforced (not required by symmetry) and full symmetry embeddings are possible without coincident nodes or zero-length links. In other cases full (Systre) symmetry embeddings are only possible with more than one node at the same point and possibly also links of zero length.

The quotient graphs presented herein were all verified by Systre (available at http://gavrog.org). The nets in Systrereadable format are available as supplementary information. ${ }^{\mathbf{5}}$

MO' $\mathrm{K}$ is supported by the World Class University program (R-31-2008-000-10055-0) and by the US National Science Foundation, grant No. DMR 1104798. DMP is supported by grant No. 14.B25.31.0005 of the Ministry of Education and Science of Russia.

\section{References}

Biggs, N. L. (1989). Ann. NY Acad. Sci. 555, 56-62. Blatov, V. A. (2006). IUCr CompComm Newsletter, 7, 4-38.

Blatov, V. A. (2007). Acta Cryst. A63, 329-343.

Blatov, V. A., Delgado-Friedrichs, O., O'Keeffe, M. \& Proserpio, D. M. (2007). Acta Cryst. A63, 418-425.

\footnotetext{
4 This graph has just 12 vertices in the quotient graph. In contrast the smallest finite graph with 3-coordinated nodes of girth 16 has 960 vertices (Biggs, 1989). 5 Supplementary material for this paper is available from the IUCr electronic archives (Reference: EO5027). Services for accessing these data are described at the back of the journal.
}

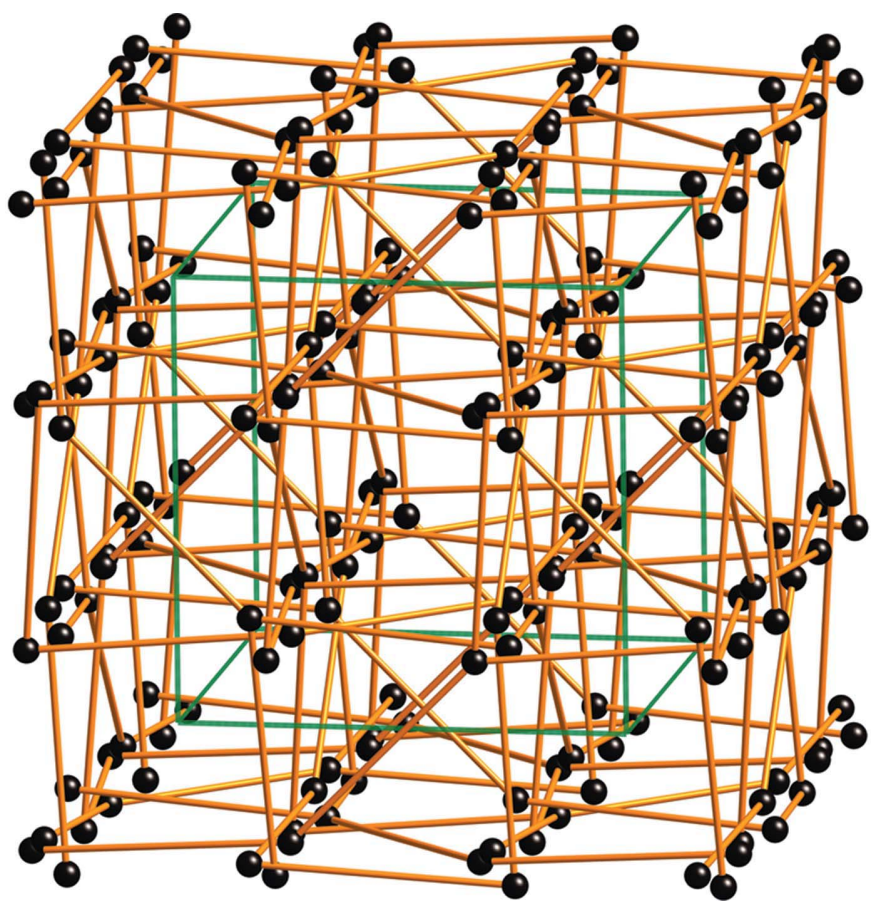

Figure 17

An embedding of the net sxt with equal links.

Blatov, V. A., O'Keeffe, M. \& Proserpio, D. M. (2010). CrystEngComm, 12, 44-48.

Bragg, W. H. \& Bragg, W. L. (1915). X-rays and Crystal Structure. London: G. Bell.

Campo, L. de, Delgado-Friedrichs, O., Hyde, S. T. \& O'Keeffe, M. (2013). Acta Cryst. A69, 483-489.

Chung, S. J., Hahn, Th. \& Klee, W. E. (1984). Acta Cryst. A40, 42-50.

Delgado-Friedrichs, O. (2004). Lecture Notes in Computer Science, 2912, 178-189.

Delgado-Friedrichs, O. (2005). Discrete Comput. Geom. 33, 67-81.

Delgado-Friedrichs, O., Foster, M. D., O’Keeffe, M., Proserpio, D. M., Treacy, M. M. \& Yaghi, O. M. (2005). J. Solid State Chem. 178, 2533-2554.

Delgado-Friedrichs, O. \& O'Keeffe, M. (2003). Acta Cryst. A59, 351360.

Delgado-Friedrichs, O. \& O'Keeffe, M. (2005). J. Solid State Chem. 178, 2480-2485.

Delgado-Friedrichs, O., O’Keeffe, M. \& Yaghi, O. M. (2007). Phys. Chem. Chem. Phys. 9, 1035-1043.

Eon, J.-G. (2007). Acta Cryst. A63, 53-65.

Eon, J.-G. (2011). Acta Cryst. A67, 68-86.

Fischer, W. (1974). Z. Kristallogr. 140, 50-74.

Grünbaum, B. (2003). Discrete and Computational Geometry: the Goodman-Pollack Festschrift, edited by B. Aronov, S. Basu, J. Pach \& M. Sharir, pp. 461-488. New York: Springer.

Grünbaum, B. (2007). Discrete Math. 307, 445-463.

Huang, X. C., Zhang, J. P., Lin, Y. Y., Yu, X. L. \& Chen, X. M. (2004). Chem. Commun. pp. 1100-1101.

Kotani, M. \& Sunada, T. (2000). Trans. Am. Math. Soc. 353, 1-20.

Liu, Y., Xu, X., Xia, Q., Yuan, G., He, Q. \& Cui, Y. (2010). Chem. Commun. 46, 2608-2610.

Liu, Y., Xu, X., Zheng, F. \& Cui, Y. (2008). Angew. Chem. Int. Ed. 47, 4538-4541.

Longuet-Higgins, H. (1963). Mol. Phys. 6, 445-460.

Ma, H., Sun, D., Zhang, L., Blatov, V. A., Guo, J. \& Sun, D. (2013). Submitted.

Montney, M. R., Mallika Krishnan, S., Patel, N. M., Supkowski, R. M. \& LaDuca, R. L. (2007). Cryst. Growth Des. 7, 1145-1153. 
Moreira de Oliveira, M. Jr \& Eon, J.-G. (2011). Acta Cryst. A67, 240251.

Moreira de Oliveira, M. Jr \& Eon, J.-G. (2013). Acta Cryst. A69, 276288.

O'Keeffe, M. (1991). Z. Kristallogr. 192, 21-37.

O'Keeffe, M. (2008). Acta Cryst. A64, 425-429.

O'Keeffe, M., Peskov, M. A., Ramsden, S. J. \& Yaghi, O. M. (2008). Acc. Chem. Res. 41, 1782-1789.

Tan, Y., Chen, H., Zhang, J., Liao, S., Dai, J. \& Fu, Z. (2012). CrystEngComm, 14, 5137-5139.
Thimm, G. (2009). Acta Cryst. A65, 213-226.

Tutte, W. T. (1960). Proc. London Math. Soc. 10, 304-320.

Tutte, W. T. (1963). Proc. London Math. Soc. 13, 743-767.

Volkringer, C., Loiseau, T., Marrot, J. \& Férey, G. (2009). CrystEngComm, 11, 58-60.

Wang, J., Li, M. \& Li, D. (2013). Chem. Sci. 4, 1793-1801.

Wells, A. F. (1977). Three-Dimensional Nets and Polyhedra. New York: Wiley.

Yu, J., Cui, Y., Wu, C., Yang, Y., Wang, Z., O'Keeffe, M., Chen, B. \& Qian, G. (2012). Angew. Chem. Int. Ed. 51, 10542-10545. 